\title{
Age-related differences in white matter diffusion measures in autism spectrum condition
}

\author{
Abigail Thompson ${ }^{1,2^{*}}$, Asal Shahidiani ${ }^{1}$, Anne Fritz ${ }^{3}$, Jonathan O'Muircheartaigh ${ }^{1,4,6}$, Lindsay Walker ${ }^{7,8}$, \\ Vera D'Almeida', Clodagh Murphy', Eileen Daly', Declan Murphy ${ }^{1,6}$, Steve Williams ${ }^{5,6}$, Sean Deoni ${ }^{7,8,9}$ \\ and Christine Ecker ${ }^{1,10}$
}

\begin{abstract}
Background: Autism spectrum condition (ASC) is accompanied by developmental differences in brain anatomy and connectivity. White matter differences in ASC have been widely studied with diffusion imaging but results are heterogeneous and vary across the age range of study participants and varying methodological approaches. To characterize the neurodevelopmental trajectory of white matter maturation, it is necessary to examine a broad age range of individuals on the autism spectrum and typically developing controls, and investigate age $\times$ group interactions.

Methods: Here, we employed a spatially unbiased tract-based spatial statistics (TBSS) approach to examine age-related differences in white matter connectivity in a sample of 41 individuals with ASC, and 41 matched controls between 7-17 years of age.

Results: We found significant age-related differences between the ASC and control group in widespread brain regions. This included age-related differences in the uncinate fasciculus, corticospinal tract, inferior longitudinal fasciculus, inferior fronto-occipital fasciculus, anterior thalamic radiation, superior longitudinal fasciculus and forceps major. Measures of fractional anisotropy (FA) were significantly positively associated with age in both groups. However, this relationship was significantly stronger in the ASC group relative to controls. Measures of radial diffusivity (RD) were significantly negatively associated with age in both groups, but this relationship was significantly stronger in the ASC group relative to controls.

Limitations: The generalisability of our findings is limited by the restriction of the sample to right-handed males with an IQ > 70. Furthermore, a longitudinal design would be required to fully investigate maturational processes across this age group.
\end{abstract}

Conclusions: Taken together, our findings suggest that autistic males have an altered trajectory of white matter maturation relative to controls. Future longitudinal analyses are required to further characterize the extent and time course of these differences.

Keywords: Autism, Tract-based spatial statistics, Diffusion weighted imaging, Connectivity

\footnotetext{
* Correspondence: Abigail.thompson@ucl.ac.uk

'Department of Forensic \& Neurodevelopmental Sciences, Institute of

Psychiatry, Psychology \& Neuroscience, King's College London, London, UK

${ }^{2}$ Developmental Change \& Plasticity Lab, Department of Psychology \&

Language Sciences, University College London, 26 Bedford Way, Bloomsbury, London WC1H OAP, UK

Full list of author information is available at the end of the article
}

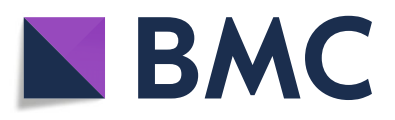

(- The Author(s). 2020 Open Access This article is licensed under a Creative Commons Attribution 4.0 International License, which permits use, sharing, adaptation, distribution and reproduction in any medium or format, as long as you give appropriate credit to the original author(s) and the source, provide a link to the Creative Commons licence, and indicate if changes were made. The images or other third party material in this article are included in the article's Creative Commons licence, unless indicated otherwise in a credit line to the material. If material is not included in the article's Creative Commons licence and your intended use is not permitted by statutory regulation or exceeds the permitted use, you will need to obtain permission directly from the copyright holder. To view a copy of this licence, visit http://creativecommons.org/licenses/by/4.0/. The Creative Commons Public Domain Dedication waiver (http://creativecommons.org/publicdomain/zero/1.0/) applies to the data made available in this article, unless otherwise stated in a credit line to the data. 


\section{Introduction}

Autism spectrum condition (ASC) is a life-long neurodevelopmental condition characterized by atypical social communication and reciprocity and a propensity for repetitive and stereotyped behaviour [1]. These principal symptoms typically manifest before the age of 2 years and are accompanied by developmental differences in brain anatomy and connectivity [2]. The neural systems underlying ASC are complex and involve alterations in multiple, spatially distributed neurocognitive systems [3]. Thus, our understanding of the neuroanatomy of ASC remains incomplete.

Despite the large phenotypic heterogeneity and complex aetiology of ASC, it is well accepted that autistic individuals show an atypical trajectory of brain development. For example, neurodevelopmental differences in brain maturation have been observed on the global level, and age-related differences in overall brain growth of total grey and white matter volume have been reported $[4,5]$. More specifically, it has been suggested that individuals with ASC undergo a period of accelerated brain growth during early postnatal life, causing the brain to be larger in early childhood relative to typically developing controls [6]. The precocious increase in total brain volume is followed by a period of atypically slow or arrested growth throughout the remainder of childhood, so that no global differences are generally observed by adulthood [5]. Increased white matter development has been suggested to contribute to early brain overgrowth in childhood [7], suggesting that brain maturation in ASC also affects the development of brain 'connectivity'.

There is evidence from structural and functional magnetic resonance imaging, positron emission tomography, and electroencephalography studies that ASC is associated with altered brain connectivity [8-11]. Atypical brain functional connectivity has been documented in both adults [12] and children with ASC $[8,13]$ and particularly in the neural networks that mediate autistic symptoms and traits. For example, altered functional connectivity of temporal-lobe networks [14] has been associated with social/emotional behaviours, and abnormalities in frontostriatal networks have been associated with ritualistic/repetitive behaviours in ASC. There is also evidence of atypical white matter structural connectivity between these regions in ASC, which has been extensively studied using diffusion tensor imaging (DTI) [15].

Diffusion imaging studies report widespread differences in white matter microstructure among infants [16], children and adolescents $[17,18]$, and adults on the autism spectrum [11, 19]. More specifically, increased mean fractional anisotropy (FA) has been reported in autistic infants and toddlers [20,21], which may indicate increased tract coherence and axonal alignment in the condition. However, this pattern seems to be reversed (i.e. lower FA values in ASC) in childhood and adolescence [22]. Some studies have also observed the opposite, with decreased FA in toddlers [23] and increased FA in later childhood [24, 25], whilst some studies have found no significant group differences in FA in children and adolescents at all [26]. Fewer differences in FA diffusion measures have been described in later adolescence and adulthood [27, 28], suggesting that between-group differences in white matter microstructure might disappear with increasing age.

Differences have also been reported in mean diffusivity (MD) and radial diffusivity (RD), but there is some disagreement. MD indicates the degree of directionindependent average diffusivity and has been shown to decrease over the course of healthy white matter maturation [29]. Studies have mainly reported no difference or higher MD values in individuals with ASC relative to controls [30, 31], although lower MD values have also been reported in one study [32]. Last, there are reports of increases in RD in both children [33] and adults with ASC [19], which may reflect changes in underlying white matter properties including reduced myelination [34].

Taken together, these findings contribute to a heterogeneous body of literature regarding diffusion imaging and ASC. This heterogeneity may indicate that the pattern of white matter abnormality varies across the investigated age range of participants and affects widespread neural systems rather than isolated brain regions. Moreover, differences in the employed methodology may affect the results. For example, the majority of DTI studies that have previously been conducted in ASC are based on a region of interest (ROI) approach [35]. ROI approaches rely on a priori hypotheses regarding the specific white matter tract or region under investigation. As it is well established that ASC is related to diffuse and spatially distributed white matter differences [3], an exploratory whole-brain approach such as TBSS is particularly well suited for examining this group of individuals.

Some studies have previously used TBSS to investigate ASC but interpretation of findings is hindered by methodological limitations and varying methodological approaches across studies. On the whole, TBSS studies have been a small scale, including a narrow age range of participants or a heterogeneous sample (including both right- and left-handed participants) [36, 37]. TBSS studies that have included the largest samples have most frequently included adults $[19,37,38]$. Studies with large sample sizes that have included children and adolescents have primarily included mixed genders [39-42], which may contribute to discrepancies across studies, due to the finding that autistic males and females may have distinct white matter developmental profiles [43]. 
Technical factors also vary across studies and may affect the results reported. Calculation of diffusion imaging parameters is affected by artefacts, which can be caused by subject-related factors such as participant motion. Another potential source of artefacts in diffusion imaging is cardiac pulsation [44]. The vast majority of studies do not report correction for this [21, 43, 45-50], suggesting that this important methodological factor has, on the whole, been overlooked. Finally, after corrections have been made, it is necessary to rotate the bmatrix to ensure accuracy of diffusion imaging parameters. Similar to cardiac gating, b-matrix rotation is largely not reported within studies $[45,47,48,51]$, suggesting this may be a contributor to the contradictory results reported across studies.

One study that did take a highly rigorous methodological approach reported reductions in FA in the autism group and a stronger developmental trajectory in the ASC group. This study included a large sample of male, right-handed ASC participants and controls who were matched for IQ (aged 10-24), corrected for motion and cardiac pulsation and rotated the b-matrix [52]. This potentially points towards a particular developmental trajectory, in which white matter development 'normalises' by adulthood in the autism group. However, as this is only one study of many that has taken such a rigorous methodological approach, more studies are needed to conclusively characterise the developmental trajectory of white matter in autism. Additionally, this study corrected for cardiac pulsation during pre-processing. A more direct approach is to use cardiac gating during acquisition. We are not aware of any TBSS study that has used cardiac gating during acquisition. Furthermore, the age range of participants included in this particular study includes the transition from adolescence to young adulthood, which is itself associated with distinct neurodevelopmental patterns [53]. Given the neurodevelopmental nature of autism, it is important for robustly designed studies with large sample sizes to focus on earlier developmental time points.

\section{Materials and methods}

\section{Participants}

Forty-one males with ASC (aged 7-17 years) and fortyone typically developing male controls (aged 8-17 years) were recruited by advertisement and assessed at the Centre for Neuroimaging Sciences, King's College London, The Institute of Psychiatry, Psychology \& Neuroscience, London. All participants were righthanded (measured using The Edinburgh Handedness inventory) [54] native English speakers and all had an IQ > 70. Exclusion criteria included pre-existing medical conditions or complications (e.g. head trauma, epilepsy), use of medication affecting brain function, a history of major psychiatric disorder (e.g. psychosis), chromosomal abnormality (e.g. fragile X, Tuberous Sclerosis, 22q11.2 deletion syndrome), and any MRI contraindications. Intellectual ability was assessed using the WASI [55]. For the autistic group, inclusion was based on an expert clinical diagnosis of autism using the International Statistical Classification of Diseases, 10th Revision (ICD-10) research criteria and confirmed using the Autism Diagnostic Interview-Revised (ADI-R) [56] (all cases reached ADI-R algorithm cutoff for autism on the domains of impaired reciprocal social interaction, communication, and repetitive behaviours and stereotyped patterns, although failure to reach cutoff for autism in a single domain by 1 point was permitted). Current symptoms were assessed using the Autism Diagnostic Observation Schedule [57], but were not used as an inclusion criterion. All participants and their parents or guardians gave informed written consent/assent in accordance with the ethics approval by the National Research Ethics Committee, Suffolk, UK.

\section{MRI}

All imaging for this study was acquired on a General Electric $3 \mathrm{~T}$ MR system. Diffusion tensor MRI scans were acquired with a spin-echo echo-planar imaging (SE-EPI) double refocused sequence providing a whole head coverage with isotropic image resolution $(2.4 \times 2.4$ $\times 2.4 \mathrm{~mm}$ ); 32 diffusion-weighted volumes with different non-collinear diffusion directions with b-factor $1300 \mathrm{sec} /$ $\mathrm{mm}^{2}$ and 6 non-diffusion-weighted volumes with 60 slices, no slice gap, TE $104.5 \mathrm{~ms}$, TR 20 R-R intervals, $128 \times 128$ acquisition matrix and FOV $=30.7 \mathrm{~cm}^{2}$ were peripherally gated (parameters compatible with [58]). Total acquisition time was approximately $12 \mathrm{~min}$. If participants were unable to tolerate scanning or obvious head movement was detected during the acquisition (due to anxiety or hyperactivity for example), they were invited to return for a second time-at which time scan quality was usually significantly improved.

\section{Preparation of data}

All data were initially visually inspected by raters at the IOPPN, KCL UK, and Brown School of Engineering, Rhode Island, to ensure inter-rater reliability. The data then went through a comprehensive correction pipeline using the TORTOISE software (https://science.nichd. nih.gov/confluence/display/nihpd/TORTOISE) [59], which registers the volumes of a DTI dataset to reduce the effects of motion and eddy current-based deformations. Corrections were performed in the native space of each subject, and appropriate rotations were applied to the b-matrix [60]. All deformations in TORTOISE were computed and applied in a single step to avoid multiple interpolations of the data. 
After correction, expert raters manually inspected the data and removed any volumes with residual artefacts. A total of ten volumes (across seven subjects) were removed, accounting for $0.3 \%$ of the total number of volumes acquired across the cohort. Differences in subject motion have been shown to be an important consideration for group comparisons of DTI data [61]. Therefore, to rule out the effect of age and between-group differences in head motion, a univariate analysis of variance was carried out to identify the main effect of age and group, as well as their interaction on the number of volumes removed and the amount of distortion and motion-correction applied during processing. For this purpose, transformation data detailing the degree of distortion and motion correction for each participant was extracted using the TORTOISE software.

\section{Estimation of the diffusion tensor}

Following the completion of quality control procedures, all subsequent analysis was carried out using the FMRIB Software Library (FSL, www.fmrib.ox.ac.uk/fsl). First, skull and non-brain tissue were removed using BET. Voxel-wise values of FA, MD and RD were then calculated.

\section{Voxel-wise analysis}

Voxel-wise statistical analysis was performed on the FA, MD and RD data using TBSS [62]. TBSS tests for between-group differences in diffusion measures across a 'skeleton' of WM tracts and across the whole brain. This procedure includes a number of steps. First, individual FA maps were non-linearly aligned to a standard space using a target image. In this study, the target image was chosen to be the most representative FA image, using the flag designation ' $-n$ '. This is the recommended option for studies of adolescents and young children. The most representative FA image (the target) was from a member of the ASC group, aged 13.5 years. After image registration, a cross-subject mean FA image was calculated, which informed the generation of the WM tract 'skeleton', thresholded at FA $>0.3$ to include major WM pathways whilst excluding peripheral tracts that are more vulnerable to partial volume effects and/or intersubject variability. Finally, each subject's FA, MD, and RD values were projected onto the group skeleton, and the resulting data was fed into voxel-wise analysis. For statistical analysis, we used the randomize function within FSL to conduct permutation-based nonparametric statistics [63] with 10,000 permutations. Areas of significant difference were displayed as a $P$ value image, where $P<0.05$, corrected for multiple comparisons across space via threshold-free cluster enhancement [64].

In order to establish the most parsimonious model of age-related differences, we initially examined linear and quadric effects with regards to age-related differences in diffusion measures. Applying a quadratic age term did not significantly increase the goodness of fit, the more parsimonious linear model was thus favoured for examining WM integrity. The GLM used in the present study therefore included a main effect of group, a linear term for age, as well as the interaction between age and group.

\section{Correlation with symptom measures}

Finally, to investigate the relationship with the severity of autistic symptom and diffusion measures, correlation analyses were conducted between the diffusion maps and ASC severity measured by the ADI-R and the ADOS. For all voxel-wise analyses, affected white matter structures were identified with the John Hopkins University white matter atlas [65].

\section{Results}

\section{Participants}

Participant demographics are listed in Table 1. Groups did not differ significantly in either age $(t(80)=0.49, p$ $=0.63)$ or IQ $(t(80)=-0.30, p=0.76)$.

\section{Movement}

There was no significant effect of age $(f(48)=0.91, p=$ $0.61)$, group $(f(1)=1.07, p=0.32)$, or group $\times$ age interaction $(f(17)=1.96, p=0.09)$ on the number of volumes removed during quality control. No significant group differences were found in the application of distortion and motion correction $(t(82)=-1.72, p=0.09)$. And no significant effect of age $(f(48)=1.76, p=0.12)$, group $(f(1)=0.87, p=0.37)$, or group $\times$ age interaction $(f(17)=1.96, p=0.08)$ on the degree of distortion and motion correction applied during processing.

\section{TBSS analysis}

The TBSS analysis did not reveal any significant between-group differences in FA, RD or MD. There were, however, significant age $\times$ group interactions for both measures of FA and RD in the UF, corticospinal tract, ILF, IFOF, anterior thalamic radiation, SLF and forceps major (Fig. 1). In these regions we found that measures of FA were significantly positively associated with age in both groups; however, this relationship was stronger in the ASC group in comparison to controls. Measures of RD were negatively associated with age in both groups; however, this relationship was stronger in the ASC group in these ROIs. The age $\times$ group interaction for measures of MD approached statistical significance, but did not reach the statistical threshold of $p<0.05$.

There were significant correlations between measures of symptom severity based on the ADOS domain of 'Stereotyped Behaviours and Restricted Interests' in spatially 
Table 1. Subject demographics. Data expressed as mean \pm standard deviation (range). There were no significant between-group differences in age $(t(80)=0.49, p=0.63)$ or IQ $(t(80)=-0.30, p=0.76)$

\begin{tabular}{lll}
\hline & ASC $(\boldsymbol{n}=\mathbf{4 1})$ & Control $(\boldsymbol{n}=\mathbf{4 1})$ \\
\hline Age (years) & $13.1 \pm 2.7$ & $12.9 \pm 2.5$ \\
& $(7-17)$ & $17)$ \\
IQ (WASI) & $110.1 \pm 15.4$ & $110.9 \pm 11.3$ \\
& $(70-140)$ & $-132)$ \\
ADI-R reciprocal social interaction & $17.7 \pm 4.4(10-26)$ & - \\
ADI-R communication & $16.3 \pm 3.8(8-23)$ & - \\
ADI-R restricted, repetitive, stereotyped behaviour & $5.5 \pm 2.3(2-11)$ & - \\
ADOS communication & $3.7 \pm 1.5(1-6)$ & - \\
ADOS reciprocal social interaction & $7.4 \pm 2.7(3-13)$ & - \\
ADOS imagination/creativity & $0.9 \pm 0.7(0-2)$ & - \\
ADOS Stereotyped behaviours, restricted interests & $1.5 \pm 1.5(0-6)$ & - \\
\hline
\end{tabular}

distributed brain regions including the UF, ILF, IFOF, corticospinal tract, and the anterior thalamic radiation (Fig. 2). There were no significant correlations with the other ADI or ADOS domains. These regions also overlap significantly with the brain regions where we observed a significant age $\times$ group interaction in the TBSS.

In order to visualise the nature of the interactions across different white matter regions, we applied region of interest masks with 16 WM regions/pathways from the John Hopkins University white matter atlas, from which diffusion measures were extracted. The masks were co-registered with the diffusion data, and mean FA, MD and RD values were obtained for each participant in each of the ROIs. Scatter plots of raw data with superimposed mean linear development trajectories for three of the sixteen WM pathways we examined are displayed in Fig. 3, for scatter plots of all 16 WM pathways refer to Supplementary Figure, 1. These are presented for illustration purposes only.

\section{Discussion}

We found significant differences in the trajectory of white matter development between male children with

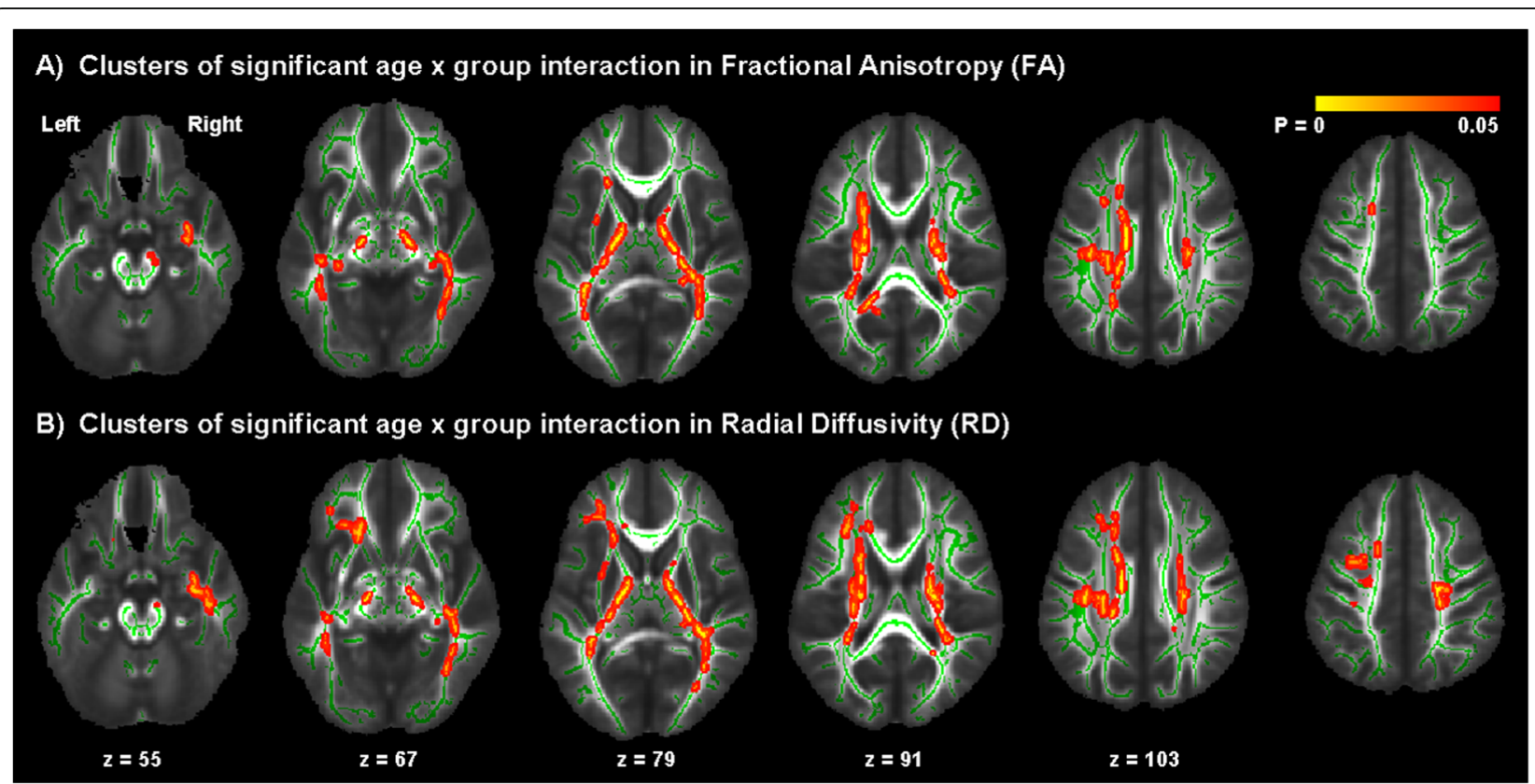

Fig. 1 Results of TBSS analysis of FA and RD. Age $\times$ group interaction for a FA and $\mathbf{b}$ RD, overlaid mean FA computed from all subjects. FA is positively associated with age in both groups but this association is stronger in the autism spectrum condition (ASC) group than in typical controls, whereas RD is negatively associated with age in both groups but this association is stronger in the ASC group than in the control group. Statistically significant voxels are displayed in red-yellow; white matter skeleton voxels are displayed in green, overlaid onto mean FA computed from all subjects. Significance was set at $p<0.05$ and was corrected for multiple comparisons with family wise error. Group differences 'thickened' and images flipped in the right-left plane for visualization purposes 


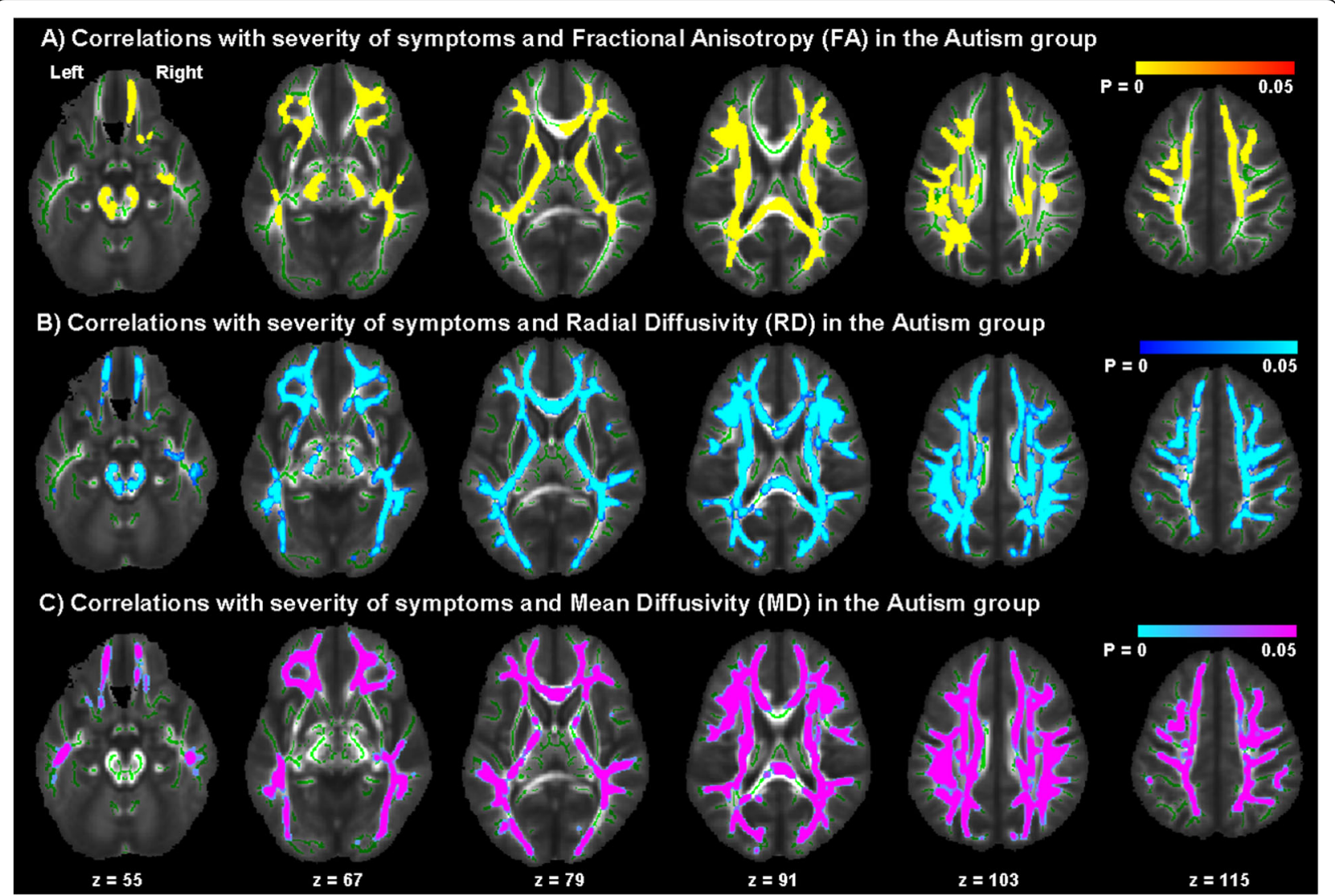

Fig. $\mathbf{2}$ Correlations with ADOS-D symptom measures. Clusters of significant correlation between the ADOS-D measures in the ASC group and $\mathbf{a}$ $F A, \mathbf{b} R D$ and $\mathbf{c} M D$. Correlations were negative for FA and positive for RD and MD. Statistically significant voxels are displayed in red, blue and purple; white matter skeleton voxels are displayed in green. Significance was set at $p<0.05$ and was corrected for multiple comparisons with family wise error. Group differences 'thickened' and images flipped in the right-left plane for visualization purposes

ASC and typically developing controls, as indicated by group differences in the relationship between diffusion measures and age. The developmental differences in white matter were observed in spatially distributed white matter regions across the brain. In both groups, measures of FA were significantly positively associated with age, while measures of both RD and MD were significantly negatively associated with age. These findings are in agreement with previous neuroimaging studies suggesting that neurodevelopmental changes in diffusivity accompany the general maturation of white matter connections in the brain [29]. We further observed that males with ASC showed a stronger positive relationship between FA with age, which was accompanied by a concomitant negative relationship with $\mathrm{MD}$ and $\mathrm{RD}$, particularly in brain regions and white matter tracts that have previously been implicated in the disorder. Additionally, we found that these DTI measures significantly correlated with the severity of stereotyped behaviours and restricted interests as measured by the ADOS (domain D) within the ASC group. On the whole, our findings corroborate previous studies that have noted widespread white matter differences in multiple brain areas in ASC and explicitly highlight the importance of consideration of age in brain development and behaviours for autistic males.

Our findings of extensive age-related between-group differences across spatially distributed white matter tracts are consistent with studies that report stronger age-related development in autism $[47,48,50,52,66]$. Some studies, however, have reported the opposite pattern, that the relationship with age is stronger in controls and smaller or absent in the ASD group [21, 36, 37, 49, 67]. These contrasting findings may relate to a number of methodological differences relating to participant inclusion criteria, such as inclusion of both genders [36, 37, 47, 48, 67] and mixed handedness $[36,37]$ as well as small sample sizes [21, 36, 47-49, 67]. Technical factors may also have contributed to contrasting results in previous studies, such as failing to correct for motion, rotate the b-matrix [21, 36, $37,43,47-49,67]$, or account for cardiac pulsation [45, $47,48,51]$. We note that only one previous study took the important methodological steps of b-matrix rotation and accounting for cardiac pulsation in the context of TBSS 
Posterior Limb of the Internal Capsule (right)
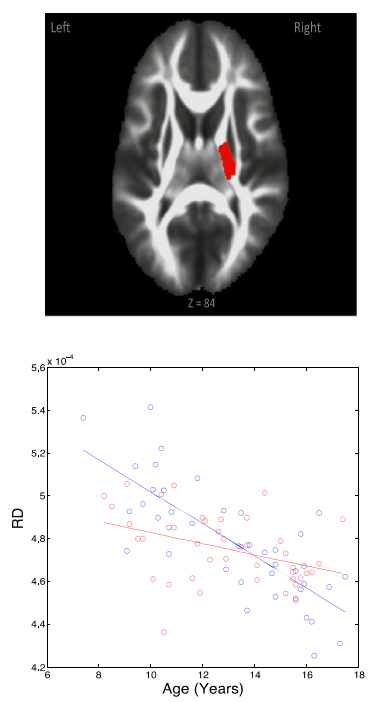
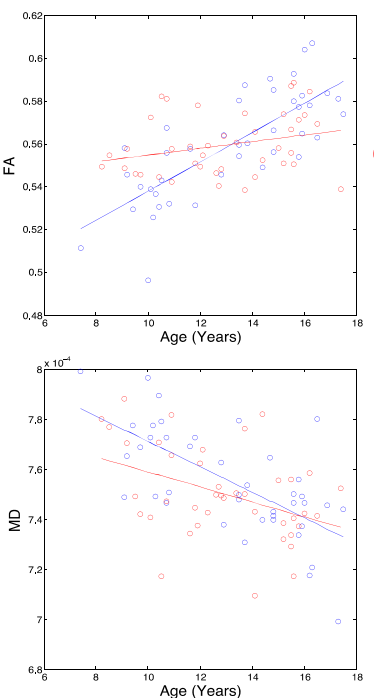
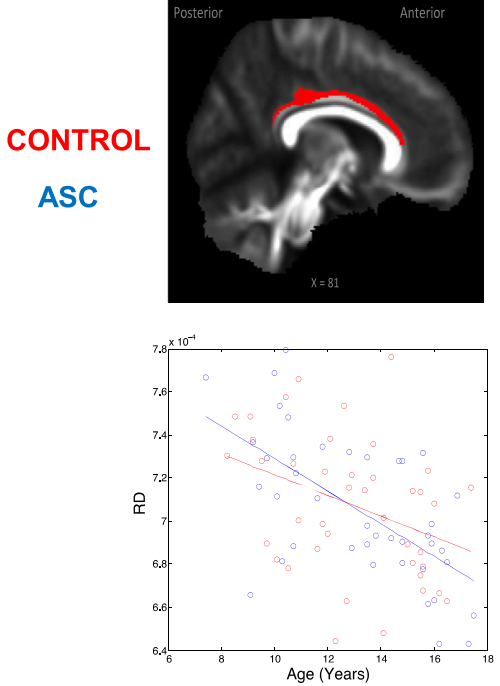

\section{Cingulum (right)}
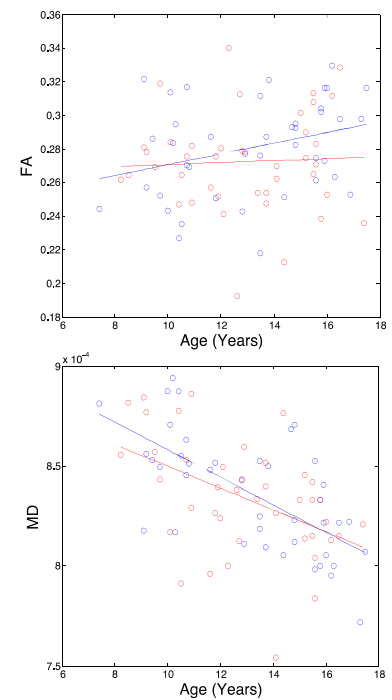

Fig. 3 Examples of age interactions in regions of interest. Examples of two regions of interest are shown for illustrative purposes (all sixteen regions of interest are included in supplementary figure 1). Regions derived from the John Hopkins University white matter atlas are co-registered with mean diffusion data. For each white matter pathway, a plot of the raw data and mean linear trend-line can be seen for FA, MD and RD. Blue corresponds to participants with ASC and red with typically-developing controls. Images flipped in the right-left plane for visualization purposes

[52] and all the others did not. In contrast to this study, we used cardiac gating during acquisition. We are not aware of any other TBSS study of autism that has done this. The age-related differences reported by this study [52] are in line with ours, with a stronger relationship in the autism group, in an older sample (aged 10-24). Our findings therefore extend the findings of Fitzgerald to a younger age group, in a robust and well-controlled study, pointing towards an altered developmental trajectory of white matter across late childhood and adolescence in autistic males.

The adaptive implications of these differences in the white matter developmental trajectory are not fully established. Some studies suggest there is 'normalisation' of white matter indices into adulthood, which may be associated with improvements of functioning [5, 52]; however, other studies report that reduced connectivity indices are associated with impairments in autistic adults [11]. In the present study, we found that lower stereotyped behaviour ADOS scores were positively associated with FA and negatively associated with RD and MD values. This trend was observed across widespread brain areas. This pattern of correlations suggests that whilst the ASC group is characterized by a stronger positive association between in FA and age, this is concomitant with a reduction of ASC symptomatology. Thus, the accelerated change in diffusion measures in the ASC group may in fact be adaptive.

Whilst we have reported extensive age-related betweengroup differences at both the voxel- and region-wise levels, we did not observe any significant between-group differences per se. This could indicate that one of the most important effects, aging, is overlooked in simple group comparisons. Some studies investigating a similar age range to ours have, in fact, reported between-group differences [68-71]; however, these have all included both males and females. As there are reported sex differences both in terms of white matter development per se [72], and in terms of white matter development in the context of autism [43, 73, 74], the discrepancy here may relate to the inclusion of both sexes. We note that in other studies across similar age ranges with exclusively male participants no between-group differences have also been reported (e.g. [75]), suggesting that an absence of betweengroup differences in males may be a consistent finding between childhood and adolescence.

It has been proposed that autism is associated with a unique trajectory of brain connectivity maturation, characterised by 'over' connectivity very early in childhood [76, 77], which then reverses over development. This leads to either 'normalisation' or, alternatively, reduced measures of white matter indices in ASC cohorts in adulthood (which explains why some studies, with all-male cohorts, including older ages report reduced measures of connectivity, e.g. [52]). If such a developmental trajectory does characterise autism, there must be a crossover point between early childhood and adulthood, at which diffusion indices between autistic individuals and controls would intersect, leading to no identifiable between-group differences. It may be the case that 
mid-late childhood and adolescence, as studied in the present cohort, constitutes such a developmental time point for ASC males. Ultimately, in a cross-sectional study such as this, we are not able to disentangle such effects. Future longitudinal studies will be required.

Overall, diffusion measures reflect a number of underlying biological processes, which need to be considered when interpreting the current findings. Alterations in FA, RD and MD may suggest differences in fibre organization and geometry, myelin formation, and myelin remodelling, as well as inflammation and gliosis [34, 78, 79]. This lack of specificity of diffusion measures constitutes an inherent limitation of DTI. This is further compounded by the finding that DTI measures may also be impacted by extrinsic factors that render groupdifferences specious. An example of such an effect comes from group differences in head motion in the scanner. Increased in-scanner motion in one group can lead to significantly decreased FA and increased RD in comparison to a relatively motionless control group [61]. These findings are particularly salient when investigating children and individuals with ASC, who may display increased movement inside the scanner. Our results, however, cannot be explained by this as the amount of inscanner motion was not found to be different between the control and ASC groups. In line with this, we did not find decreased FA and increased RD in the group comparison, which would have been predicted as a result of increased motion [61].

\section{Limitations}

The generalisability of our findings is limited by the restriction of the sample to right-handed males with an IQ $>70$. Thus, it is not possible to determine the applicability of these findings to autistic females, or individuals with an IQ of $<70$, which constitutes a substantial proportion of autistic individuals. Furthermore, the crosssectional nature of the study means that the increased heterogeneity associated with ASC cannot be accounted for and also limits our understanding of maturational processes across this age group, for which a future study using a longitudinal design would be required.

\section{Conclusions}

To conclude, using a rigorous methodological approach, our findings indicate a distinct neurodevelopmental trajectory of white matter development in young males with ASC. However, without further histological validation, these differences cannot be attributed to a specific biological process or feature. As the differences we see in FA, MD and RD are widespread, our study confirms that ASC is a neural systems disorder with neurodevelopmental differences in white matter brain connectivity.

\section{Supplementary information}

Supplementary information accompanies this paper at https://doi.org/10. 1186/s13229-020-00325-6.

Additional file 1. Supplementary figure. Results of region of interest analysis.

\section{Abbreviations}

ADI-R: Autism Diagnostic Interview-Revised; ADOS: Autism Diagnostic Observation Schedule; ASC: Autism spectrum condition; BET: Brain extraction tool; DTI: Diffusion tensor imaging; FA: Fractional anisotropy; FMRIB: Oxford Center for functional MRI of the brain; FSL: FMRIB Software Library; GLM: General linear model; ICD-10: International Classification of Diseases-10; IFOF: Inferior fronto-occipital fasciculus; ILF: Inferior longitudinal fasciculus; IOPPN: Institute of Psychiatry, Psychology \& Neuroscience; IQ: Intelligence quotient; KCL: King's College London; MD: Mean diffusivity; MRI: Magnetic resonance imaging; RD: Radial diffusivity; ROI: Region of interest;

SLF: Superior longitudinal fasciculus; TBSS: Tract-based spatial statistics;

UF: Uncinate fasciculus; UK: United Kingdom; WASI: Wechsler Abbreviated Scale of Intelligence; WM: White matter

\section{Acknowledgements}

The research leading to these results has received support from (1) the Medical Research Council (MRC, UK) G0800298 awarded to Doctor Sean CL Deoni, (2) the Medical Research Council (MRC, UK) G0400061 awarded to Professor Declan Murphy, (3) the Dr. Mortimer and Theresa Sackler Foundation, (4) the MRC AIMS Consortium, a collaboration of autism research centres in the UK including the Institute of Psychiatry, London, The Autism Research Centre, University of Cambridge and the Autism Research Group, University of Oxford. It is funded by the Medical Research Council (MRC) UK and headed by the Department of Forensic and Neurodevelopmental Sciences, Institute of Psychiatry. The Consortium members are in alphabetical order: Bailey AJ, Baron-Cohen S, Bolton PF, Bullmore ET, Carrington S, Chakrabarti B, Daly EM, Deoni SC, Ecker C, Happe F, Henty J, Jezzard P, Johnston P, Jones DK, Lombardo M, Madden A, Mullins D, Murphy CM, Murphy DG, Pasco G, Sadek S, Spain D, Steward R, Suckling J, Wheelwright S and Williams SC. and (5) EU-AIMS (European Autism Interventions)/EU AIMS-2-TRIALS, a European Innovative Medicines Initiative Joint Undertaking under Grant Agreements No. 115300 and 777394, the resources of which are composed of financial contributions from the European Union's Seventh Framework Programme (Grant FP7/2007-2013). JOM is supported by a Sir Henry Dale Fellowship jointly funded by the Welcome Trust and the Royal Society (Grant Number 206675/Z/17/Z). We would like to thank the National Institute for Health Research (NIHR) Biomedical Research Centre at South London and Maudsley NHS Foundation Trust and King's College London for their ongoing support.

\section{Authors' contributions}

AT, AS, JO'M, LW, CM, ED, DM, SD and CE were responsible for the study design. AT, AS and VD'A were responsible for the data collection, AT, AS and CE were responsible for the statistical analysis. AT, AS, AF and CE were responsible for the manuscript preparation. Statistical analysis was completed by Abigail Thompson and Asal Shahidiani under the supervision of Christine Ecker. The authors read and approved the final manuscript.

\section{Funding}

Financial support from this work has been provided by (1) the Medical Research Council (MRC, UK) G0800298 and (2) the Medical Research Council (MRC, UK) G0400061.

\section{Availability of data and materials}

The authors have full access to all the data supporting the conclusions of this article, which will be shared on an open access repository, and should the manuscript be accepted for publication.

\section{Ethics approval and consent to participate}

All participants and their parents or guardians gave informed written consent/assent in accordance with ethics approval by the National Research Ethics Committee, Suffolk, UK. 


\section{Consent for publication}

Not applicable.

\section{Competing interests}

The authors declare that they have no competing interests.

\section{Author details}

'Department of Forensic \& Neurodevelopmental Sciences, Institute of Psychiatry, Psychology \& Neuroscience, King's College London, London, UK. 2Developmental Change \& Plasticity Lab, Department of Psychology \& Language Sciences, University College London, 26 Bedford Way, Bloomsbury, London WC1H OAP, UK. ${ }^{3}$ The Centre for Research in Autism and Education (CRAE), Psychology and Human Development, UCL, London, UK. ${ }^{4}$ Centre for the Developing Brain, Department of Perinatal Imaging and Health, St. Thomas' Hospital, King's College London, London, UK. ${ }^{5}$ Department of Neuroimaging, Institute of Psychiatry, Psychology \& Neuroscience, King's College London, London, UK. ${ }^{6}$ MRC Centre for Neurodevelopmental Disorders, King's College London, London, UK. ${ }^{7}$ Advanced Baby Imaging Lab, Hasbro Childrens Hospital, Providence, RI, USA. ${ }^{8}$ Pediatrics and Radiology, Warren Alpert medical school, Brown University, Providence, USA. ${ }^{9}$ Maternal, Newborn \& Child Health Discovery \& Tools at the Bill and Melinda Gates Foundation, Seattle, USA. ${ }^{10}$ Department of Child and Adolescent Psychiatry, Psychosomatics and Psychotherapy, University Hospital, Goethe University Frankfurt am Main, Deutschordenstrasse 50, 60528 Frankfurt am Main Germany.

\section{Received: 7 June 2019 Accepted: 3 March 2020}

Published online: 18 May 2020

\section{References}

1. Wing $L$. The autistic spectrum. Lancet. 1997:350:1761

2. Geschwind DH, Levitt P. Autism spectrum disorders: developmental disconnection syndromes. Curr Opin Neurobiol. 2007.

3. Ecker C, Spooren W, Murphy DGM. Translational approaches to the biology of autism: false dawn or a new era. Mol Psychiatry. 2013.

4. Carper RA, Moses P, Tigue ZD, Courchesne E. Cerebral lobes in autism: early hyperplasia and abnormal age effects. Neuroimage. 2002

5. McAlonan GM, Cheung V, Cheung C, Suckling J, Tai KS, Lam GY, et al. Mapping the brain in autism. A voxel-based MRI study of volumetric differences and intercorrelations in autism. Brain. 2005;128:268.

6. Courchesne E, Karns C, Davis H, Ziccardi R, Carper R, Tigue Z, et al. Unusual brain growth patterns in early life in patients with autistic disorder: an MRI study. Neurology. 2001

7. Herbert MR. Ziegler DA. O'Brien LM, Lange N, Bakardjiev A, et al. Dissociations of cerebral cortex, subcortical and cerebral white matter volumes in autistic boys. Brain: Deutsch CK; 2003 126: 1182

8. Di Martino A, Kelly C. Grzadzinski R, Mennes M, Mairena MA, et al. Aberrant striatal functional connectivity in children with autism. Biol Psychiatry: Zuo XN; 2011 69:847.

9. Castelli F. Autism. Brain: Asperger syndrome and brain mechanisms for the attribution of mental states to animated shapes; 2002.

10. Carson AM, Salowitz NMG, Scheidt RA, Dolan BK, Van Hecke AV. Electroencephalogram coherence in children with and without autism spectrum disorders: decreased interhemispheric connectivity in autism. Autism Res. 2014;7:334

11. Thompson A, Murphy D, Dell'Acqua F, Ecker C, McAlonan G. Howells H, et al. Impaired communication between the motor and somatosensory homunculus is associated with poor manual dexterity in autism spectrum disorder. Biol Psychiatry 2017.

12. Just MA, Cherkassky VL, Keller TA, Minshew NJ. Cortical activation and synchronization during sentence comprehension in high-functioning autism: evidence of underconnectivity. Brain. 2004;127:1811.

13. Dinstein I, Pierce K, Eyler L, Solso S, Malach R. Behrmann M, et al. Neuron: Disrupted neural synchronization in toddlers with autism; 2011.

14. Adolphs R, Sears L, Piven J. Abnormal processing of social information from faces in autism. J Cogn Neurosci. 2001;13:232.

15. Pierpaoli C. Jezzard P., Basser PJ, Barnett A, Di Chiro G. Diffusion tensor MR imaging of the human brain. Radiology: 1996.

16. Wolff JJ, Gu H, Gerig G, Elison JT, Styner M, Gouttard S, et al. Differences in white matter fiber tract development present from 6 to 24 months in infants with autism. Am J Psychiatry. 2012.
17. Weinstein M, Ben-Sira L. Levy $Y$, Zachor DA, Itzhak E Ben, Artzi M, et al. Abnormal white matter integrity in young children with autism. Hum Brain Mapp; 2011

18. Walker L, Gozzi M, Lenroot R, Thurm A, Behseta B, Swedo S, et al. Diffusion tensor imaging in young children with autism: biological effects and potential confounds. Biol Psychiatry. 2012

19. Catani M, Dell'Acqua F, Budisavljevic S, Howells H. Thiebaut De Schotten M, Froudist-Walsh $\mathrm{S}$, et al. Frontal networks in adults with autism spectrum disorder. Brain: 2016

20. Solso S, Xu R, Proudfoot J, Hagler DJ, Campbell K, Venkatraman V, et al. Diffusion tensor imaging provides evidence of possible axonal overconnectivity in frontal lobes in autism spectrum disorder toddlers. Biol Psychiatry. 2016;79:676.

21. Ouyang M, Cheng H, Mishra V, Gong G, Mosconi MW, Sweeney J, et al. Atypical age-dependent effects of autism on white matter microstructure in children of 2-7 years. Hum Brain Mapp. 2016;37(2):819-32.

22. Im WY, Ha JH, Kim EJ, Cheon KA, Cho J, Song DH. Impaired white matter integrity and social cognition in high-function autism: diffusion tensor imaging study. Psychiatry Investig. 2018.

23. Zhang L, Li K, Zhang C, Qi X, Zheng N, Wang G. Arcuate fasciculus in autism spectrum disorder toddlers with language regression. Open Med. 2018:13:90.

24. Fitzgerald J, Leemans A, Kehoe E, O'Hanlon E, Gallagher L, McGrath J. Abnormal fronto-parietal white matter organisation in the superior longitudinal fasciculus branches in autism spectrum disorders. Eur J Neurosci. 2018:47:652.

25. Li Y, Fang H, Zheng W, Qian L, Xiao Y, Wu Q, et al. A fiber tractography study of social-emotional related fiber tracts in children and adolescents with autism spectrum disorder. Neurosci Bull. 2017:36:722.

26. Failla MD, Peters BR, Karbasforoushan H, Foss-Feig JH, Schauder KB, Heflin $\mathrm{BH}$, et al. Intrainsular connectivity and somatosensory responsiveness in young children with ASD. Mol Autism. 2017;8:25

27. Gibbard CR, Ren J. Skuse DH, Clayden JD Clark CA. Structural connectivity of the amygdala in young adults with autism spectrum disorder. Hum Brain Mapp 2018.

28. Braden BB, Smith CJ. Thompson A. Glaspy TK, Wood E, Vatsa D, et al. Executive function and functional and structural brain differences in middleage adults with autism spectrum disorder. Autism Res 201710:1945.

29. Schmithorst VJ, Wilke M, Dardzinski BJ, Holland SK. Correlation of white matter diffusivity and anisotropy with age during childhood and adolescence: a cross-sectional diffusion-tensor MR imaging study. Radiology. 2002.

30. Groen WB, Buitelaar JK, van der Gaag RJ, Zwiers MP. Pervasive microstructural abnormalities in autism: a DTI study. J Psychiatry Neurosci. 2011.

31. Karahanoğlu FI, Baran B, Nguyen QTH, Meskaldji DE, Yendiki A, Vangel M, et al. Diffusion-weighted imaging evidence of altered white matter development from late childhood to early adulthood in autism spectrum disorder. Neurolmage Clin 2018.

32. Mengotti P, D'Agostini S, Terlevic R, De Colle C, Biasizzo E, Londero D, et al. Altered white matter integrity and development in children with autism: a combined voxel-based morphometry and diffusion imaging study. Brain Res Bull. 2011.

33. Shukla DK, Keehn B, Lincoln AJ, Müller RA. White matter compromise of callosal and subcortical fiber tracts in children with autism spectrum disorder: a diffusion tensor imaging study. J Am Acad Child Adolesc Psychiatry. 2010.

34. Song SK, Sun SW, Ramsbottom MJ, Chang C, Russell J, Cross AH. Dysmyelination revealed through MRI as increased radial (but unchanged axial) diffusion of water. Neuroimage. 2002.

35. Travers BG, Adluru N, Ennis C, Tromp DPM, Destiche D, Doran S, et al. Diffusion tensor imaging in autism spectrum disorder: a review. Autism Res. 2012.

36. Shukla DK, Keehn B, Müller RA. Tract-specific analyses of diffusion tensor imaging show widespread white matter compromise in autism spectrum disorder. J Child Psychol Psychiatry Allied Discip. 2011;52:286.

37. Koolschijn PCMP, Caan MWA, Teeuw J, Olabarriaga SD, Geurts HM. Agerelated differences in autism: the case of white matter microstructure. Hum Brain Mapp. 2017;38:82.

38. Itahashi T, Yamada T, Nakamura M, Watanabe H, Yamagata B, Jimbo D, et al. Linked alterations in gray and white matter morphology in adults with high-functioning autism spectrum disorder: a multimodal brain imaging study. Neurolmage Clin. 2015;7:155.

39. Lisiecka DM, Holt R, Tait R. Ford M, Lai MC, Chura LR, et al. Developmental white matter microstructure in autism phenotype and corresponding endophenotype during adolescence. Transl Psychiatry; 2015. 
40. Carper RA, Treiber JM, DeJesus SY, Müller RA. Reduced hemispheric asymmetry of white matter microstructure in autism spectrum disorder. J Am Acad Child Adolesc Psychiatry 2016.

41. Vogan VM, Morgan BR, Leung RC, Anagnostou E, Doyle-Thomas K, Taylor MJ. Widespread white matter differences in children and adolescents with autism spectrum disorder. J Autism Dev Disord. 2016;46(6):2138-47.

42. Solders SK, Carper RA, Müller RA. White matter compromise in autism? Differentiating motion confounds from true differences in diffusion tensor imaging. Autism Res. 2017;10(10):1606-20.

43. Lei J, Lecarie E, Jurayj J, Boland S, Sukhodolsky DG, Ventola P, et al. Altered neural connectivity in females, but not males with autism: preliminary evidence for the female protective effect from a quality-controlled diffusion tensor imaging study. Autism Res. 2019;12(10):1472-83.

44. Bopp MHA, Yang J, Nimsky C, Carl B. The effect of pulsatile motion and cardiac-gating on reconstruction and diffusion tensor properties of the corticospinal tract. Sci Rep. 2018;8:1

45. Vogan VM. Morgan BR, Leung RC, Anagnostou E, Doyle-Thomas K, Taylor MJ. Widespread white matter differences in children and adolescents with autism spectrum disorder. J Autism Dev Disord 2016

46. Libero LE, Burge WK, Deshpande HD, Pestilli F, Kana RK. White matter diffusion of major fiber tracts implicated in autism spectrum disorder. Brain Connect. 2016;6(9):691-9.

47. Ameis SH, Fan J, Rockel C, Voineskos AN, Lobaugh NJ, Soorya L, et al. Impaired structural connectivity of socio-emotional circuits in autism spectrum disorders: a diffusion tensor imaging study. PLoS One. 2011.

48. Bakhtiari R, Zürcher NR, Rogier O, Russo B, Hippolyte L, Granziera C, et al. Differences in white matter reflect atypical developmental trajectory in autism: a tract-based spatial statistics study. Neurolmage Clin. 2012.

49. Cheng Y, Chou KH, Chen IY, Fan YT, Decety J, Lin CP. Atypical development of white matter microstructure in adolescents with autism spectrum disorders. Neuroimage [Internet]. 2010;50(3):873-882Available from: https:// doi.org/10.1016/j.neuroimage.2010.01.011

50. Kleinhans NM, Pauley G, Richards T, Neuhaus E, Martin N, Corrigan NM, et al. Age-related abnormalities in white matter microstructure in autism spectrum disorders. Brain Res [Internet]. 2012;1479:1-16. Available from: https://doi.org/10.1016/j.brainres.2012.07.056.

51. Perkins TJ, Stokes MA, McGillivray JA, Mussap AJ, Cox IA, Maller JJ, et al. Increased left hemisphere impairment in high-functioning autism: a tract based spatial statistics study. Psychiatry Res Neuroimaging [Internet]. 2014; 224(2):119-123Available from: https://doi.org/10.1016/j.pscychresns.2014.08. 003.

52. Fitzgerald J, Gallagher L, McGrath J. Widespread disrupted white matter microstructure in autism spectrum disorders. J Autism Dev Disord. 2019; 49(7):2664-74

53. Taber-Thomas B, Perez-Edgar K. Emerging adulthood brain development. In: the Oxford handbook of emerging adulthood. 2016

54. Oldfield RC. The assessment and analysis of handedness: the Edinburgh inventory. Neuropsychologia. 1971;9:97.

55. Wechsler D. Wechsler abbreviated scale of intelligence. NY.: Psychol Corp Harcourt Brace Company New York; 1999.

56. Lord C, Rutter M, Le Couteur A. Autism diagnostic interview-revised: a revised version of a diagnostic interview for caregivers of individuals with possible pervasive developmental disorders. J Autism Dev Disord. 1994;5: 659.

57. Lord C, Risi S, Lambrecht L, Cook EH, Leventhal BL, Dilavore PC, et al. The autism diagnostic observation schedule-generic: a standard measure of social and communication deficits associated with the spectrum of autism. J Autism Dev Disord. 2000;30:205.

58. Langen M, Leemans A, Johnston P, Ecker C, Daly E, Murphy CM, et al. Fronto-striatal circuitry and inhibitory control in autism: findings from diffusion tensor imaging tractography. Cortex. 2012.

59. Pierpaoli C, Walker L. TORTOISE: an integrated software package for processing of diffusion MRI data. ... Process Diffus .... 2010;

60. Leemans A. Jones DK. The B-matrix must be rotated when correcting for subject motion in DTI data. Magn Reson Med; 2009.

61. Koldewyn K, Yendiki A, Weigelt S, Gweon H, Julian J, Richardson H, et al. Differences in the right inferior longitudinal fasciculus but no general disruption of white matter tracts in children with autism spectrum disorder. Proc Natl Acad Sci U S A. 2014;111:1981.
62. Smith SM, Jenkinson M, Johansen-Berg H, Rueckert D, Nichols TE, Mackay CE, et al. Tract-based spatial statistics: voxelwise analysis of multi-subject diffusion data. Neuroimage. 2006.

63. Nichols TE, Holmes AP. Nonparametric permutation tests for functional neuroimaging: a primer with examples. Hum Brain Mapp. 2002.

64. Smith SM, Nichols TE. Threshold-free cluster enhancement: addressing problems of smoothing, threshold dependence and localisation in cluster inference. Neuroimage. 2009.

65. Mori S, Oishi K, Jiang H, Jiang L, Li X, Akhter K, et al. Stereotaxic white matter atlas based on diffusion tensor imaging in an ICBM template. Neuroimage; 2008

66. McLaughlin K, Travers BG, Dadalko OI, Dean DC, Tromp D, Adluru N, et al. Longitudinal development of thalamic and internal capsule microstructure in autism spectrum disorder. Autism Res. 2018;11(3):450-62.

67. Mak-Fan KM, Morris D, Vidal J, Anagnostou E, Roberts W. Taylor MJ. White matter and development in children with an autism spectrum disorder. Autism; 2013.

68. Fishman I, Datko M, Cabrera Y, Carper RA, Müller RA. Reduced integration and differentiation of the imitation network in autism: a combined functional connectivity magnetic resonance imaging and diffusionweighted imaging study. Ann Neurol. 2015;78(6):958-69.

69. Bos DJ, Merchán-Naranjo J, Martínez K, Pina-Camacho L, Balsa I, Boada L, et al. Reduced gyrification is related to reduced interhemispheric connectivity in autism spectrum disorders. J Am Acad Child Adolesc Psychiatry. 2015;54(8):668-76.

70. Carper RA, Solders S, Treiber JM, Fishman I, Müller RA. Corticospinal tract anatomy and functional connectivity of primary motor cortex in autism. J Am Acad Child Adolesc Psychiatry. 2015;54(10):859-67.

71. Nair A, Carper RA, Abbott AE, Chen CP, Solders S, Nakutin S, et al. Regional specificity of aberrant thalamocortical connectivity in autism. Hum Brain Mapp. 2015;36(11):4497-511.

72. Hsu JL, Leemans A, Bai CH, Lee CH, Tsai YF, Chiu HC, et al. Gender differences and age-related white matter changes of the human brain: a diffusion tensor imaging study. Neuroimage. 2008;39(2):566-77.

73. Zeestraten EA, Gudbrandsen MC, Daly E, De Schotten MT, Catani M Dell'Acqua F, et al. Sex differences in frontal lobe connectivity in adults with autism spectrum conditions. Transl Psychiatry. 2017;7(4):e1090.

74. Nordahl CW, losif AM, Young GS, Perry LM, Dougherty R, Lee A, et al. Sex differences in the corpus callosum in preschool-aged children with autism spectrum disorder. Mol Autism [Internet]. 2015;6(1):1-11. Available from: ???

75. Duerden EG, Card D, Roberts SW, Mak-Fan KM, Chakravarty MM, Lerch JP, et al. Self-injurious behaviours are associated with alterations in the somatosensory system in children with autism spectrum disorder. Brain Struct Funct. 2014:6:1251.

76. Andrews DS, Lee JK, Solomon M, Rogers SJ, Amaral DG, Nordahl CW. A diffusion-weighted imaging tract-based spatial statistics study of autism spectrum disorder in preschool-aged children. J Neurodev Disord 2019; 11(1):1-12.

77. Hazlett HC, Gu H, Munsell BC, Kim SH, Styner M, Wolff JJ, et al. Early brain development in infants at high risk for autism spectrum disorder. Nature: 2017

78. Beaulieu $\mathrm{C}$. The basis of anisotropic water diffusion in the nervous system a technical review. NMR Biomed. 2002.

79. O'Muircheartaigh J. Dean DC, Ginestet CE, Walker L, Waskiewicz N, Lehman $\mathrm{K}$, et al. White matter development and early cognition in babies and toddlers. Hum Brain Mapp: ; 201435:4475.

\section{Publisher's Note}

Springer Nature remains neutral with regard to jurisdictional claims in published maps and institutional affiliations. 